\title{
Estimation of Reaction, Diffusion and Restitution Parameters for a 3D Myocardial Model Using Optical Mapping and MRI
}

\author{
J. Relan ${ }^{1}$, M. Pop ${ }^{2}$, H. Delingette ${ }^{1}$, \\ G.A. Wright ${ }^{2}$, N. Ayache ${ }^{1}$, and M. Sermesant ${ }^{1,3}$ \\ ${ }^{1}$ INRIA, Asclepios Project, Sophia Antipolis, France \\ ${ }^{2}$ University of Toronto, Sunnybrook Health Sciences Centre, Canada \\ ${ }^{3}$ King's College London, Division of Imaging Sciences, St. Thomas Hospital, UK
}

\begin{abstract}
Personalisation, i.e. parameter estimation of a cardiac ElectroPhysiology (EP) model is needed to build patient-specific models, which could then be used to understand the true complex dynamics involved in patient's pathology. In this paper, we present a personalisation method for a simplified ionic 3D EP model, the Mitchell-Schaeffer model. The personalisation is performed by optimising the 3D model parameters, which represent the tissue conductivity, Action Potential Duration (APD) and restitution for APD and conduction velocity, using only $2 \mathrm{D}$ epicardial surface data obtained ex-vivo from optical imaging of large porcine healthy hearts. We are also able to estimate all of the model parameters, thus resulting in a total heart-specific 3D EP model. Finally, we also test the sensitivity of the described personalisation results with respect to different pacing locations.
\end{abstract}

\section{Introduction}

Modelling of the cardiac electrophysiology has been an important research interest for the last decades, but in order to translate this work into clinical applications, there is an important need for personalisation of such models, i.e. estimation of the model parameters which best fit the simulation to the clinical data. Cardiac model personalisation is required to develop predictive models that can be used to improve therapy planning and guidance. For instance, RadioFrequency (RF) ablation therapy on patients suffering from Ventricular Tachycardia (VT) has a success rate of only $50 \%$ due to non availability of a clinical consensus on the optimum RF ablation patterns [1. Thus the procedure is a trial and error process and highly dependent on cardiologist's experience. Personalised cardiac models can provide a way to test in silico different RF ablation patterns, consecutively increasing the success rate of RF therapy.

A variety of mathematical models describing the cardiac electrophysiology have been developed and simulated at various scales. These models can be broadly categorised into three main categories: Ionic Models (IM), Phenomenological Models (PM) and Eikonal Models (EM). IM [2] characterise ionic currents flowing through the cardiac cell membrane and have a lot of parameters 
and variables, thus are not well suited to solve the inverse problem. EM [3] are very simple, describing only the time at which a depolarisation wave reaches a given point without precisely modelling the action potential. At the intermediate level are PM [4], which describe the action potential generation and propagation along the cell membrane. Here, we personalise a simplified generic ionic model, the Mitchell-Schaeffer (MS) model [5], modelling the action potential as a combination of sodium $\left(\mathrm{Na}^{+}\right)$, calcium $\left(\mathrm{Ca}^{2+}\right)$ and potassium $\left(\mathrm{K}^{+}\right)$phenomenological ionic currents.

In the past years, authors have focused on the parameter estimation of the PM and MS model on 3D volumes 6/7/8/9 using optical and MR data. In these works, the tissue conductivity parameter was estimated only on 17 American Heart Association (AHA) subdivisions of the bi-ventricular myocardium. Also not all model parameters were estimated. The two main contributions of this paper are: 1) Estimation of the tissue conductivity parameter using a multiresolution technique, and 2) Estimation of all model parameters, simulating tissue features such as local Conduction Velocity (CV), Action Potential Duration (APD) and APD and CV restitutions. Thus resulting in a total heart-specific 3D electrophysiology model.

\section{3D Electrophysiology Model Simulation}

The MS model [5] is a generic simplified ionic model and is described by the following system of Partial Differential Equations (PDE):

$$
\left\{\begin{array}{l}
\partial_{t} u=\operatorname{div}(D \nabla u)+\frac{z u^{2}(1-u)}{\tau_{\text {in }}}-\frac{u}{\tau_{\text {out }}}+J_{\text {stim }}(t) \\
\partial_{t} z= \begin{cases}\frac{(1-z)}{\tau_{\text {open }}} & \text { if } z<z_{\text {gate }} \\
\frac{-z}{\tau_{\text {close }}} & \text { if } z>z_{\text {gate }}\end{cases}
\end{array}\right.
$$

where $u$ is the normalised action potential variable, and $z$ is the gating variable for $\mathrm{Na}^{+}$influx, which depicts the repolarisation phase. $J_{\text {in }}=\left(z u^{2}(1-u)\right) / \tau_{\text {in }}$ represents the combination of inward $\mathrm{Na}^{+} \& \mathrm{Ca}^{2+}$ phenomenological ionic currents and $J_{\text {out }}=-u / \tau_{\text {out }}$ represents the outward $\mathrm{K}^{+}$phenomenological ionic current. $J_{\text {stim }}$ is the stimulation current at the pacing location. The parameter $\tau_{\text {in }}$ mostly depicts the cardiac tissue conductivity for the model reaction part. $\tau_{\text {in }}$ and $\tau_{\text {out }}$ jointly controls the shape of CV and APD restitutions. The parameter $\tau_{\text {open }}$ mainly controls the slope of the APD restitution curves and $\tau_{\text {close }}$ is directly related to the asymptotic value of the APD restitution curves.

The diffusion term in the model is controlled by the diffusion tensor $D$. This spatial diffusion can be related to a pseudo-conductivity of the cardiac tissue. In the longitudinal direction of the fiber, this pseudo-conductivity is set to a parameter $d$ and to $d / 2.5^{2}$ in the transverse directions [4]. The MS model is spatially integrated using a linear tetrahedral mesh of the bi-ventricular myocardium, taking into account the fiber orientation as well, and is temporally 

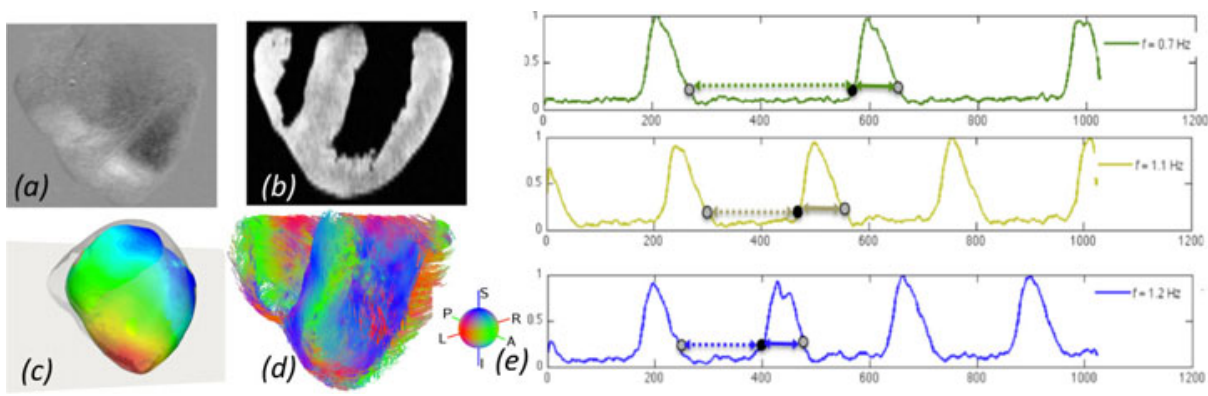

Fig. 1. (a) A snapshot from $2 \mathrm{D}+\mathrm{t}$ optical mapping data recorded for the electrical activity (antero-lateral view), (b) A slice from DT-MRI volume acquired for geometry and fiber orientations, (c) The optical and MR fusion showing the stereoscopic surface (transparent) fused with the volumetric mesh along with projection of the extracted features (here depolarisation time isochrones) from the optical data to the volumetric mesh, (d) Tracking of fibers from DT-MRI, (e) The filtered optical signals for a pixel of dataset 1 showing the $A P D-D I$ pairs for various pacing frequencies.

integrated using an optimum time integration scheme (MCNAB), which was tested in details in [107]. The electrical time-step used here is $\delta t=0.1 \mathrm{~ms}$ on a mesh with a mean edge length of $h=1.5 \mathrm{~mm}$.

\section{Optical and MR Dataset Processing}

In this paper, we performed the adjustments on a healthy porcine heart model. The experimental set-up is described in details in [11. The acquired data consists of Diffusion Tensor MRI (DT-MRI) representing geometry and fiber orientation (Fig 1 (b \& d)), and epicardial optical recordings (Fig 1 (a \& e)), from which we compute features (such as the Depolarisation Time (DT) isochrones, the APD maps and the $\mathrm{CV}$ and APD restitution). The features are then projected on the volumetric myocardial mesh derived from the DT-MRI (Fig 1(c)) as explained in [6. This results in features on the epicardial surface of the 3D biventricular model. Here, we used 2 ex-vivo hearts, which were MR and optically imaged for steady state cycles. The datasets were prepared as follows: one heart was optically imaged to produce 3 different optical datasets, all paced at one location (at the apex of the LV epicardium), but for 3 different pacing frequencies (Fig 1(e) \& Fig 2):

\section{- Dataset 1: $0.7 \mathrm{~Hz}, 1.1 \mathrm{~Hz}$ and $1.2 \mathrm{~Hz}$}

Second heart was optically imaged to produce 2 different optical datasets, paced at a frequency of $1.1 \mathrm{~Hz}$, but obtained using 2 different pacing locations (Fig 2) Near the apex of:

- Dataset 2: Right Ventricle Epicardium (RV-Epi) and Left Ventricle Endocardium (LV-Endo). 

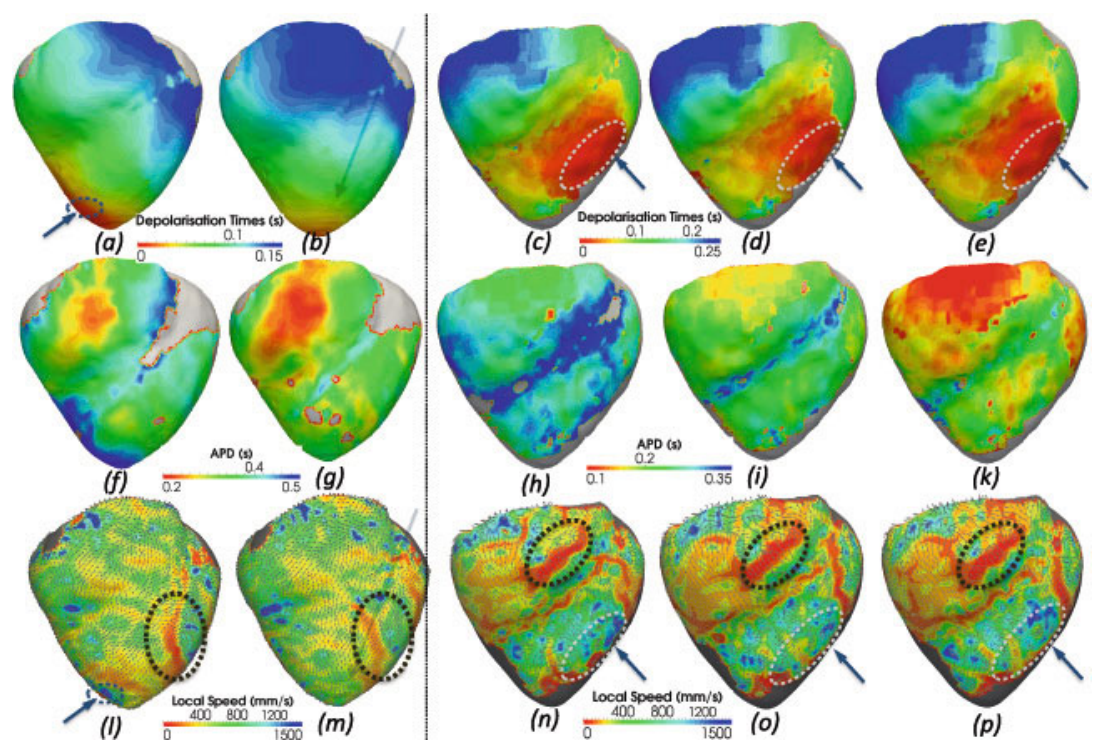

Fig. 2. The first row shows epicardial DT isochrones, second row shows the APD maps and third row shows the estimated local conduction velocity depicting the tissue conductivity. First two columns are for dataset 2 and remaining three columns are for dataset 1 . Arrows and white dotted contours highlight the pacing locations and black dashed contours highlight areas of low tissue conductivity.

Although these were healthy hearts, we could identify discrete areas of low conductivity (see black dashed contour in Fig 2). This was most likely due to tissue becoming ischemic around a small collateral blood vessel, partially occluded by an air bubble accidentally trapped into the perfusion line, resulting in oxygen deprivation of the tissue and further installation of acute ischemia and cellular uncoupling.

\section{Personalisation Method}

Estimation of the model parameters that result in a simulation which is similar to the measured data is defined as personalisation. Here, we perform model personalisation by matching features such as the DT isochrones, the APD maps and the $\mathrm{CV}$ and $\mathrm{APD}$ restitution all derived from the optical data. Dataset 2 was used for personalisation using DT isochrones and APD maps only, whereas the dataset 1 was used for personalisation using additional features such as APD and CV restitutions.

\subsection{Feature: Depolarisation Times}

The epicardial DT isochrones show variations in CV of the propagating action potential wave over the epicardial surface. The local conduction velocity $C V^{m s d}$ 
is estimated from the spatial gradient of these measured DT isochrones $T$ on the epicardial surface as, $1 / C V^{m s d}=\left\|\nabla T_{x}\right\|$ [4. Due to the imaging and registration errors, the DT isochrones could present local variations. To avoid the amplification of this noise by the spatial derivatives of the DT, we smooth $C V^{m s d}$ by averaging it over a neighbouring area, weighted by the Euclidean distance between the vertices and the point where the speed is computed. This feature is used for estimation of parameter $d$, which is achieved in the following two successive phases:

Calibration: This step is used to initialise the model parameter values using analytical relationships between the measures and the parameters. The calibration function used here is similar to [7] and is given as $c(d)=\alpha \sqrt{d}+\beta$, where $c$ is the $\mathrm{CV}$ and the constants $\alpha$ and $\beta$ are determined by performing several model simulations for a range of $d$ values and computing the corresponding $c$, and then fitting the function in a non-linear least squares sense to the measures $c$. Once the relationship is estimated, it is used to determine the initial parameter value $d$ for the median value of $c$ computed for the actual reference data.

Iterative Adjustment: This step is used to optimise the parameter $d$ locally in space, using a multi-resolution technique, with calibration result as an initial guess. In order to start the domain decomposition, we divide the left ventricle into 17 zones as defined by AHA and a similar division of 9 zones for the right ventricle is performed. The algorithm used here is a trust region method [12] and is implemented using the Trilinos solver package 1 . Here we use an objective function that minimises the sum of squared differences (SSD) between the simulated and the measured depolarisation times by iteratively adjusting the $d$ parameter value for each zone. Thereafter, when the SSD error remains stagnant, we perform another domain decomposition level where zones of higher error are divided into 4 (Fig 4) and so on. In order to have smoother connectivity between zone parameters and to avoid piecewise parameter functions, we estimate parameter $d$ on the zonal barycenter and perform a diffusive parameter regularisation as explained in the next subsection.

\subsection{Feature: Action Potential Duration}

For $\tau_{\text {close }}$, the maximum APD for a single cardiac cycle is directly given by the model [5] as follows: $A P D=\tau_{\text {close }} l n\left(1 / h_{\text {min }}\right)$ where $h_{\text {min }}=4 \tau_{\text {in }} / \tau_{\text {out }}$. For dataset 2 , we only have one measured APD available from the data, we chose to adjust $\tau_{\text {close }}$, while keeping the other parameter values from the literature $[5]$. It is defined by the model that $c$ has no relationship with $\tau_{c l o s e}$, which provides no coupling between the APD and the CV. Thus we can simultaneously adjust parameter $d$ and $\tau_{\text {close }}$. The defined relationship between $\tau_{\text {close }}$ and APD remains valid also in $3 \mathrm{D}$ thus allowing us to directly estimate it locally at each vertex. The parameter $\tau_{\text {close }}$ is estimated on the epicardial surface having measures. To propagate the $\tau_{\text {close }}$ values from the epi to endocardium, we diffuse the $\tau_{\text {close }}$

\footnotetext{
${ }^{1}$ http://trilinos.sandia.gov/
} 

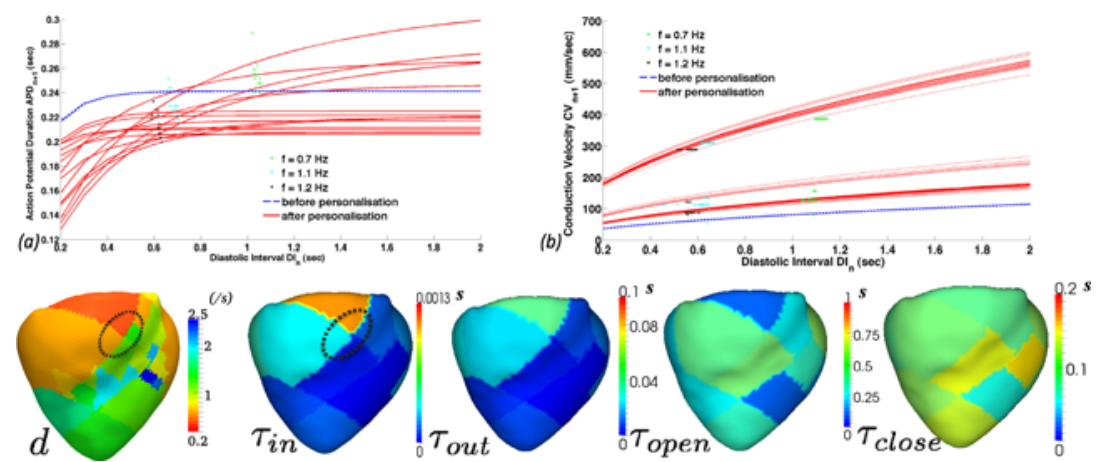

Fig. 3. For dataset 1, (a) shows the fitting of the model restitution to the extracted APD restitution points from different pacing frequency data for a few number of pixels. (b) is the same but for CV restitution. Lower row shows the estimated parameter vector $\theta$, with parameter $d$ estimated using multi-resolution.

spatially in the myocardium using $\operatorname{div}(\nabla P)=0$ in myocardium with $P=\tau_{\text {close }}$ estimated on epicardium. The same is done to diffuse parameter $d$ with $P=d$ estimated at the zonal barycenter as explained before.

\subsection{Feature: CV and APD Restitution}

Restitution defines the dependency of the next cycle APD (resp. CV) on the previous cycle Diastolic Interval (DI). For a constant pacing frequency $f$, a steady state Basic Cycle Length (BCL) remains constant : $B C L=1 / f=A P D+D I$ and thus $A P D-D I$ relationship remains constant. In order to extract the observed macroscopic restitution, we need to have the heart optically imaged for multiple pacing frequencies, thus resulting in multiple BCL (Fig 1(e)) and multiple $A P D-D I$ pairs for a spatial point (pixel). Thus this personalisation step was performed only on Dataset 1 . APD restitution curve for MS model is explicitly formulated [5] as

$$
f(D I)=A P D=\tau_{\text {close }} \ln \left(\frac{h(D I)}{h_{\min }}\right)
$$

where $h(D I)=1-\left(1-h_{m i n}\right) e^{-D I / \tau_{o p e n}}$, with $f(D I)=A P D$ is the succeeding APD and $D I$ is the preceding DI. Similarly also CV restitution curve is derived [5] as

$$
g(D I)=\left(\frac{1}{4}\left(1+\sqrt{1-\frac{h_{\min }}{h(D I)}}\right)-\frac{1}{2}\left(1-\sqrt{1-\frac{h_{\min }}{h(D I)}}\right)\right) \sqrt{\frac{2 d h(D I)}{\tau_{\text {in }}}}
$$

with $g(D I)=C V$ as the succeeding CV. From Eq 2 \& Eq 3, we can observe parameter ratio $(h(D I))$ controlling both APD \& CV restitution. This shows a coupling between both restitutions. Thus we chose to estimate the parameters 

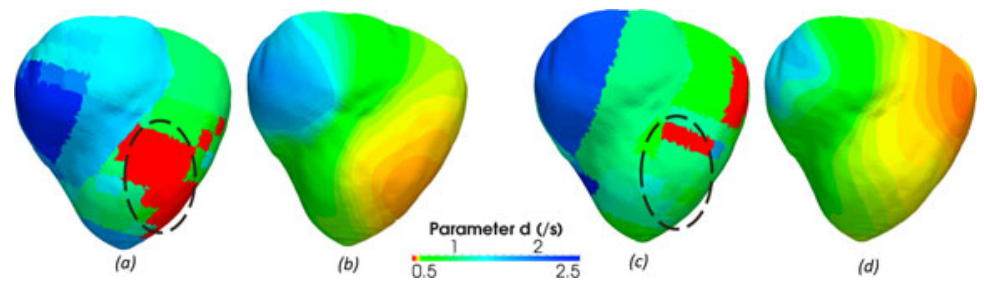

(d)
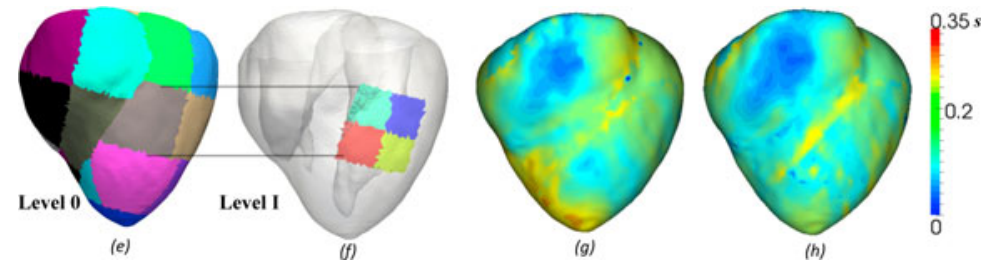

Fig. 4. For dataset 2, (a) is the final domain decomposition for parameter $d$ after personalisation for RV-Epi and (b) parameter $d$ after diffusive regularisation, whereas (c) \& (d) are the same but for LV-Endo, (e) \& (f) levels for the multi-resolution method (Level 0 represents the AHA subdivision, Level I is the subdivision of a zone into 4), (g) \& (h) are the parameter $\tau_{\text {close }}$ maps for RV-Epi and LV-Endo (respectively).

for $\mathrm{CV}$ restitution $\left(h_{\text {min }}, \tau_{\text {in }}, d\right)$ and APD restitution $\left(h_{\text {min }}, \tau_{\text {open }}, \tau_{\text {close }}\right)$ in a joint manner, by having a cost function $C_{r}$ which minimises the error on both restitution curves. $C_{r}$ is given as

$$
C_{r}=\min _{\theta} \sum_{j=1}^{N}\left(\left(f\left(D I^{j}, \theta\right)-A P D^{j}\right)^{2}+\left(g\left(D I^{j}, \theta\right)-C V^{j}\right)^{2}\right)
$$

where $N$ is the total number of frequency data, $A P D^{j}$ (resp. $C V^{j}$ ) is the measured $A P D$ (resp. $C V$ ) for the preceding measured $D I$ at the optical data pixel, and $\theta=\left[\tau_{\text {close }}, h_{\text {min }}, \tau_{\text {open }}, \tau_{\text {in }}\right]$ is the estimated parameter vector. $\theta$ is estimated locally at each pixel of the optical data and then a mean value for each AHA zone is computed and set to the zonal barycenter in the mesh. The parameter optimisation used here is a constrained Active-Set Algorithm, which uses a sequential quadratic programming method. The parameter $d$ is then adjusted using iterative adjustment for a single cycle at the lowest pacing frequency $(f=0.7$ $\mathrm{Hz}$ ), since it represents the asymptotic value of the $\mathrm{CV}$ restitution curve. This is done in order to have the parameter $d$ take into account the CV due to the wave front curvature on the volumetric mesh.

\section{Results}

\subsection{Application to Dataset 1}

The model is personalised to dataset 1 by estimating the parameter vector $\theta$ (explained in section 4.3), using restitution defined with steady state cycles from 


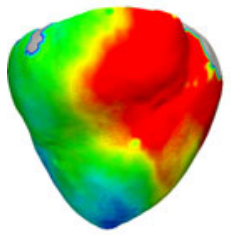

(a)

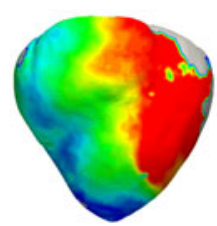

(g)

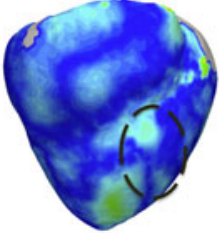

(b)

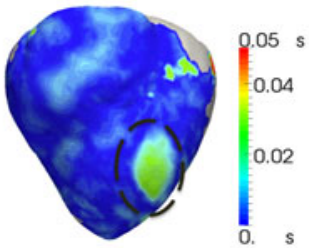

(h)

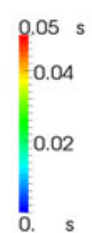

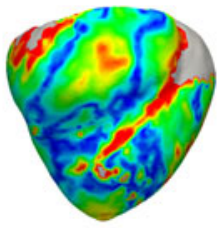

(d)

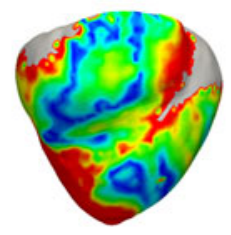

(j)

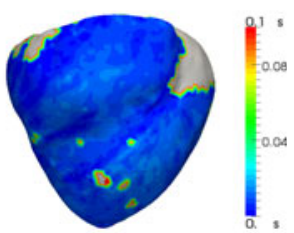

(e)

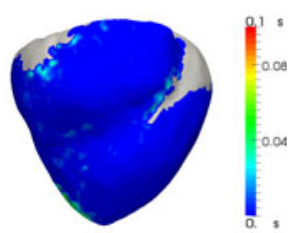

(k)

Fig. 5. For dataset 2, first row stands for LV-Endo and the second row for RV-Epi. DT error maps before $(\mathrm{a}, \mathrm{g})$ and after $(\mathrm{b}, \mathrm{h})$ personalisation. APD error maps before $(\mathrm{d}, \mathrm{j})$ and after $(\mathrm{e}, \mathrm{k})$ personalisation.

different pacing frequencies. Before personalisation, the model is simulated using standard parameter values 5 . The absolute mean square error $C_{r}(\mathrm{Eq} 4 \mathrm{4})$, before personalisation is 20.35 reduced to 0.54 after personalisation, which implies a good fit of the both APD and CV restitution curves to the data, as shown in Fig 3 (a \& b). The zonal parameters estimated show clear differences in values of $\tau_{\text {in }} \& \tau_{\text {out }}$ for $\mathrm{LV}$ and RV. $\tau_{\text {close }}$ shows lower values at the pacing location and RV zones, thus showing APD heterogeneity between the LV and RV. $\tau_{\text {open }}$, a parameter controlling the APD restitution slope, shows lower values (flat slope) near the pacing and basal regions compared to the remaining epicardium. The parameters depicting the tissue conductivity from the diffusion term $(d)$ and reaction term $\left(\tau_{i n}\right)$, are also able to locate the low conductivity area observed in the dataset 1 (see black dashed contour in Fig 2 \& 3). Also parameter $d$ shows an overall map of low conductivity over the heart, compared to dataset 2 (see Fig. 3 \& 4), which is confirmed by higher total activation time $(\approx 250 \mathrm{~ms})$ for this dataset.

\subsection{Application to Dataset 2}

The model is personalised to dataset 2 using DT isochrones and APD maps for two different pacing locations as explained before. A comparison of the estimated parameter values and APD and DT errors after personalisation for both pacing locations, helps us also evaluate the sensitivity of the described personalisation method. As the electrophysiology personalisation is performed on the same heart but under different pacing scenarios, we should expect the personalisation results to be similar, showing low sensitivity of the personalisation method to different pacing locations. 


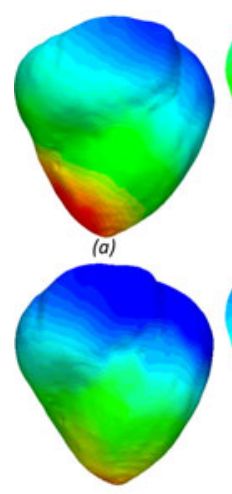

(e)

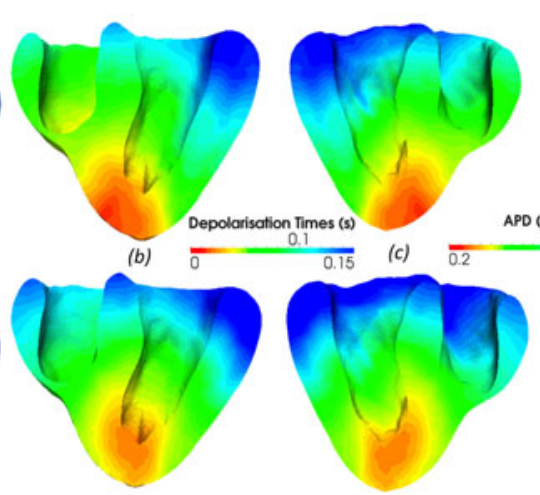

(f)

(g)

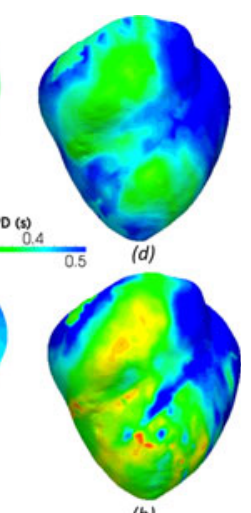

(h)

Fig. 6. For dataset 2, simulated volumetric DT isochrones (a,b,c) and APD maps (d) for LV-Endo, after personalisation. Similarly for RV-Epi (e,f,g \& h) after personalisation. (For true experimental DT \& APD values refer Fig 2)

Comparison of the Estimated Parameter Values and Errors: From DT error maps of two different pacing locations (Fig 5 (b) and $5(\mathrm{~h})$ ), we can observe that the personalisation method does reduce the overall error with both pacing locations. It is also able to locate the areas of low $\mathrm{CV}$ irrespective of the pacing location (black dashed contour in Fig 2 \& 5). APD error maps also show less error with both pacing locations (Fig $5(\mathrm{e})$ and $5(\mathrm{k})$ ). This demonstrates low sensitivity of the personalisation method application to different pacing locations. Fig 4 and Table 1 show a qualitative and quantitative comparison of the estimated parameter $d$ for both pacing locations. Here we can observe that the parameter values are mostly similar for both pacing locations, except for pacing location LV endocardium, where the low conductivity area is moved more towards the base as probably the purkinje system is recruited (activated) using the endocardial pacing location thus increasing the conduction velocity. On the other hand, the locally estimated parameter $\tau_{\text {close }}$ for both pacing locations is very similar as shown in Fig 4 and Table 1 . This analysis does confirms the low sensitivity of the estimated parameter values to various pacing locations.

Table 1. Comparison of the estimated parameter values and the mean errors for personalisation using dataset 2. (Mean- $\Delta$, Standard Deviation- $\sigma$ )

\begin{tabular}{|c|c|c|c|c|c|c|}
\hline \multirow[t]{2}{*}{$\begin{array}{l}\text { Pacing } \\
\text { Location }\end{array}$} & \multicolumn{2}{|c|}{ Parameter $d \pm \sigma\left(s^{-1}\right)$} & \multirow[t]{2}{*}{$\begin{array}{l}\text { DT Error } \\
\Delta \pm \sigma \\
\quad(\mathrm{ms})\end{array}$} & \multicolumn{2}{|c|}{$\begin{array}{c}\text { Parameter } \\
\tau_{\text {close }} \pm \sigma \times 10^{-4} \\
(\mathrm{~ms})\end{array}$} & \multirow[t]{2}{*}{$\begin{array}{c}\text { APD } \\
\text { Error } \\
\Delta \pm \sigma \\
(\mathrm{ms})\end{array}$} \\
\hline & $\mathrm{LV}$ & $\mathrm{RV}$ & & LV & $\mathrm{RV}$ & \\
\hline LV-Endo & $0.95 \pm 0.03$ & $1.36 \pm 0.16$ & $4.22 \pm 6.75$ & $0.22 \pm 1.25$ & $0.20 \pm 4.90$ & $4.98 \pm 8.89$ \\
\hline RV-Epi & $0.96 \pm 0.03$ & $1.38 \pm 0.11$ & $2.54 \pm 5.12$ & $0.22 \pm 3.04$ & $0.21 \pm 6.81$ & $4.73 \pm 5.57$ \\
\hline
\end{tabular}




\section{Discussion and Conclusion}

In this work, we described a personalisation method for a simplified ionic MS model in 3D, using epicardial activation maps obtained with optical imaging. The model personalisation is done using the measured CV, APD and their restitution properties. The estimated pseudo-conductivity parameter $d$ map is able to locate low conductivity areas for both datasets, using a multi-resolution technique. All the model parameters can be estimated when data is acquired at multiple frequencies and thus exhibits the restitution properties. Next, we evaluated the sensitivity of our personalisation method to different pacing scenarios and observed the reproduction of the same estimated parameter values irrespective of the pacing location. Such fairly robust cardiac electrophysiology personalisation with different pacing locations from epi- or endocardium opens up possibilities for clinical applications, where typically only the endocardial surface can be mapped and with often an ambiguity on the pacing location. Nevertheless, we also need to validate the model predictions, at different heart rates and pacing locations, which is important in arrhythmias. This is the topic which will be addressed in the next step.

\section{Acknowledgements}

The research leading to these results was partially funded by the the euHeart project (FP7/2007-2013 under grant agreement n 224495).

\section{References}

1. Aliot, E., Stevenson, W., Almendral-Garrote, J.: Ehra/hrs expert consensus on catheter ablation of ventricular arrhythmias. Europace 11, 771-817 (2009)

2. Noble, D.: A modification of the Hodgkin-Huxley equations applicable to Purkinje fibre action and pace-maker potentials. Physiology 160, 317-352 (1962)

3. Sermesant, M., Konukoglu, E., Delingette, H., Coudiere, Y., Chinchaptanam, P., Rhode, K., Razavi, R., Ayache, N.: An anisotropic multi-front fast marching method for real-time simulation of cardiac electrophysiology. In: Sachse, F.B., Seemann, G. (eds.) FIHM 2007. LNCS, vol. 4466, pp. 160-169. Springer, Heidelberg (2007)

4. Keener, J., Sneyd, J.: Mathematical Physiology. Springer, Heidelberg (1998)

5. Mitchell, C.C., Schaeffer, D.G.: A two current model for the dynamics of cardiac membrane. B. of Mathematical Biology 65, 767-793 (2003)

6. Lepiller, D., Sermesant, M., Pop, M., Delingette, H., Wright, G.A., Ayache, N.: Cardiac electrophysiology model adjustment using fusion of $\mathrm{mr}$ and optical imaging. In: Metaxas, D., Axel, L., Fichtinger, G., Székely, G. (eds.) MICCAI 2008, Part I. LNCS, vol. 5241, pp. 678-685. Springer, Heidelberg (2008)

7. Relan, J., Sermesant, M., Delingette, H., Pop, M., Wright, G.A., Ayache, N.: Quantitative comparison of two cardiac electrophysiology models using personalisation to optical and mr data. In: ISBI, pp. 1027-1030 (2009) 
8. Relan, J., Sermesant, M., Pop, M., Delingette, H., Sorine, M., Wright, G., Ayache, N.: Parameter estimation of a $3 \mathrm{~d}$ cardiac electrophysiology model including the restitution curve using optical and $\mathrm{mr}$ data. In: IFMBE Proceedings of World Congress on Medical Physics and Biomedical Engineering, pp. 1716-1719 (2009)

9. Relan, J., Sermesant, M., Pop, M., Delingette, H., Sorine, M., Wright, G., Ayache, N.: Volumetric prediction of cardiac electrophysiology using a heart model personalised to surface data. In: MICCAI Workshop on Cardiovascular Interventional Imaging and Biophysical Modelling - CI2BM 2009, pp. 19-27 (2009)

10. Ethier, M., Bourgault, Y.: Semi-implicit time discretization schemes for the bidomain model. Siam J. Numer. Anal. 46(5), 2443-2468 (2008)

11. Pop, M., Sermesant, M., Lepiller, D., Truong, M.V., McVeigh, E.R., Crystal, E., Dick, A., Delingette, H., Ayache, N., Wright, G.A.: Fusion of optical imaging and mri for the evaluation and adjustment of macroscopic models of cardiac electrophysiology: A feasibility study. Med. Image Anal. (July 2008)

12. Conn, A.R., Gould, N.I.M., Toint, P.: Trust Region Methods. SIAM, Philadelphia (2000) 